\section{Neuroimaging abnormalities and seizure recurrence in a prospective cohort study of Zambians with human immunodeficiency virus and first seizure}

Michael J. Potchen,1 Omar K. Siddiqi,,2,3 Melissa A. Elafros, 4 Igor J. Koralnik, ${ }^{3}$ William H. Theodore, 5

Izukanji Sikazwe, ${ }^{6}$ Lisa Kalungwana,7 Christopher M. Bositis, 8

Gretchen L. Birbeck 9,10

${ }^{1}$ Neuroradiology Division, Department of Imaging Sciences, University of

Rochester, NY, USA; 2Department of Internal Medicine, University of Zambia, Lusaka, Zambia; ${ }^{3}$ Division of NeuroVirology, Beth Israel Deaconess Medical Center, Boston, MA, USA; ${ }^{4}$ College of Human Medicine, Michigan State University, East Lansing, MI, USA; ${ }^{5}$ Clinical Epilepsy Section, United States National Institutes of Health, Bethesda, MD, USA; ${ }^{6}$ Centre for Infectious Disease Research in Zambia, Lusaka, Zambia; 7Department of Psychology, University of Zambia, Lusaka, Zambia; ${ }^{8}$ Greater Lawrence Family Health Center, Lawrence, MA, USA; ${ }^{9}$ Epilepsy Division, Department of Neurology, University of Rochester, NY, USA; ${ }^{10}$ Chikankata Epilepsy Care Team, Mazabuka, Zambia

\section{Abstract}

In HIV-positive individuals with first seizure, we describe neuroimaging findings, detail clinical and demographic risk factors for imaging abnormalities, and evaluate the relationship between imaging abnormalities and seizure recurrence to determine if imaging abnormalities predict recurrent seizures. Among 43 participants (mean 37.4 years, 56\% were male), 16 (37\%) were on antiretroviral drugs, 32 (79\%) had advanced HIV disease, and (28) $66 \%$ had multiple seizures and/or status epilepticus at enrollment. Among those with cerebrospinal fluid studies, 14/31 (44\%) had opportunistic infections (OIs). During follow-up, 9 (21\%) died and 15 (35\%) experienced recurrent seizures. Edema was associated with OIs (odds ratio: 8.79; confidence interval: 1.03-236) and subcortical atrophy with poorer scores on the International HIV Dementia Scale) (5.2 vs. 9.3; $\mathrm{P}=0.002)$. Imaging abnormalities were not associated with seizure recurrence or death $(\mathrm{P}>0.05)$. Seizure recurrence occurred in at least a third and over $20 \%$ died during follow-up. Imaging was not predictive of recurrent seizure or death, but imaging abnormalities may offer additional diagnostic insights in terms of $\mathrm{OI}$ risk and cognitive impairment.

\section{Introduction}

In patients presenting with their first seizure, identifiable structural brain abnormalities are a risk factor for recurrent seizure and imaging helps guide clinicians in determining whether or not to recommend the initiation of chronic treatment with antiepileptic drugs (AEDs) after an initial seizure. ${ }^{1}$ The epidemiologic data that informs this decisionmaking process was obtained in studies of HIV negative individuals. Whether structural brain lesions in people with HIV, a significant proportion of which may represent transient, infectious phenomena, are predictive of recurrent seizures and indicate the need for chronic therapy is unknown. In resource-limited settings, where enzyme-inducing AEDs may be the only available medications, initiating longterm epilepsy treatment carries the additional concern of adverse AED-antiretroviral (ARV) interactions and warrants especially careful consideration. ${ }^{2}$

In the US, neuroimaging is indicated in HIV+ patients with new onset seizure for diagnostic purposes and to assure that lumbar puncture is not contraindicated. ${ }^{3}$ In subSaharan African (SSA) countries, HIV remains the number one cause of disease and disability, despite the availability of ARVs. ${ }^{4}$ Although neuroimaging was not routinely available in most African settings in 2004, ${ }^{5}$ there is some evidence that, at least in tertiary care centers, access to imaging technology is improving. ${ }^{6}$

There are few studies of HIV-related neuroimaging findings in Africa, ${ }^{7}$ and viral clade differences may make extrapolations from the United States and European studies inappropriate. $^{8}$ In African children with HIV, brain atrophy with dilatation of the lateral ventricles, calcification of the basal ganglia, and periventricular white matter involvement are the most common neuroimaging findings. ${ }^{7}$ We report the neuroimaging findings in a Cohort study of HIV-Associated Seizures and Epilepsy (CHASE study) which was conducted in Zambia. Acute clinical and demographic risk factors for imaging abnormalities and the prognostic value of imaging for seizure recurrence and death are also described.
Correspondence: Gretchen L. Birbeck, Epilepsy Division, Department of Neurology, University of Rochester, 265 Crittenden Blvd, Rochester, NY 14624, USA.

Tel.: + 1.585.273.4265 - Fax: +1.517.507.5440.

E-mail gretchen_birbeck@urmc.rochester.edu

Key words: prognosis, seizure recurrence, opportunistic infection, computed tomography, cognitive impairment, CHASE.

Acknowledgements: the authors would thank our UTH-based research support staff and administrators for their dedicated, tireless efforts to support this work.

Contributions: MJP, first draft, imaging data interpretation, study design, manuscript revision; OKS, clinical follow-up of patients, infectious work up, laboratory support, study design, manuscript revision; MAE, study design, patients follow up, manuscript revision; IJK, study design, infectious evaluations, manuscript revision; WHT, study design, manuscript revision; IS, clinical follow-up of patients, study design, manuscript revision; LK, neuropsychiatric assessments, study design, manuscript revision; CMB, study design, manuscript revision; GLB, study design and analysis, manuscript revision.

Funding: the project described was supported by the Fogarty International Center and the National Institute of Neurological Disorders and Stroke (NINDS) under Award Number 1R21NS073509. The content is solely the responsibility of the authors and does not necessarily represent the official views the National Institutes of Health. MAE was supported in part by an MD/PhD Fellowship from Spectrum Health, the AMA Foundation, and the Lois C. Walker Endowed Fund for Student Research in the Michigan State University College of Human Medicine. OKS was supported by an American Academy of Neurology Clinical Research Fellowship. IJK is supported in part by NINDS R01 047029 and 074995 . WHT was supported by the International League against Epilepsy grant for Partnership's between North American Epilepsy Centers and Africa's. International League against Epilepsy's.

Conflict of interests: the authors declare no potential conflict of interests.

Received for publication: 13 July 2014. Accepted for publication: 11 August 2014.

This work is licensed under a Creative Commons Attribution NonCommercial 3.0 License (CC BYNC 3.0).

(C) Copyright M.J. Potchen et al., 2014 Licensee PAGEPress, Italy

Neurology International 2014; 6:5547 doi:10.4081/ni.2014.5547 


\section{Materials and Methods}

HIV+ adults who presented to the University Teaching Hospital in Lusaka, Zambia with new onset seizure between August 1, 2011 and June 19, 2013 were enrolled in CHASE. Additional inclusion criteria included age $\geq 18$ years and no prior history of seizures except for childhood febrile seizures. Written consent from the patient or their proxy was required. At enrollment, clinical and demographic characteristics were obtained as well as an electroencephalograph and, for patients who consented to lumbar puncture, cerebrospinal fluid (CSF) analysis which included extensive studies using PCR technology to identify opportunistic infections (OIs). After discharge, neuropsychiatric assessments with instruments previously used in Zambia to detect HIV-associated neurocognitive disorders (HAND) and psychiatric morbidity 9,10 were conducted by a neuropsychologist (LK) including the Shona Symptom Questionnaire (SSQ), ${ }^{11}$ the International HIV Dementia Scale (IHDS) and the Zambian Mini-mental State Exam (zMMSE). ${ }^{12-14}$ CHASE participants were followed prospectively with explicit assessments made during their HIV clinic visits to identify recurrent seizures. Death outcomes were obtained through clinic and hospital records and contact with family members through December 21, 2013. When family members were interviewed after the death of a participant, specific questions were asked regarding recurrent seizures including seizures around the time of death.

A head computed tomography (CT) with contrast was performed as part of the study for CHASE participants in whom no seizure etiology was established based upon other diagnostic assessments, including CSF analyses. CT imaging was acquired on a Siemens CT2007YS CT scanner and magnetic resonance (MR) imaging on a Siemen's Magnetom Essenza 1.5T MRI scanner Siemens, Munich, Germany). CT protocols included: $1.5 \mathrm{~mm}$ contiguous axial imaging from the foramen magnum through the vertex for 3D reconstruction with $4 \mathrm{~mm}$ oblique axial imaging pre- and postcontrast. CT contrast using Ultravist 300 was administered. MRI protocols included sagittal T1, Axial T2, FLAIR, Diffusion weighted imaging with apparent diffusion coefficients, and T1 pre and post contrast, as well as Coronal T2 images. MRI contrast using Magnevist was administered by hand injection. Several participants had neuroimaging studies, including 2 MRIs, obtained as part of their routine clinical care. In these patients, there were no predetermined criteria for contrast administration and not all of them received contrast, possibly due to medical contraindications or cost incurred directly by the patient. Both CT and MRI findings were interpreted and coded using an early version of NeuroInterp which codes dichotomous or ordinal values for specific anatomical findings. 15 These included the presence of cortical abnormalities, the presence of white matter abnormalities, the extent of white matter involvement, deep structural abnormalities, and posterior fossa abnormalities. Other radiographic findings captured included the presence of: abnormal masses or mass effect, abnormal fluid collections, intracranial bleeds, and calcifications. The extent of ventricular size (decreased, normal or increased), and brain volume (on a scale of 1-5 with 3 being normal) were determined as well as contrast enhancement, if contrast was administered. The study radiologist (MJP) interpreted both the clinical and research images. He was provided with age and gender and was aware of the patients' HIV status and presenting symptoms, but was blinded to other clinical findings and outcome. Data were analyzed using Epi Info version 7.0.

We used chi-square, t-test analysis or the Kruskal-Wallis test where population variances were non-homogenous to i) identify clinical and demographic risk factors for imaging findings, and ii) determine if imaging findings were predictive of death or recurrent seizure. The small sample size precluded multivariate analyses. A P-value $<0.05$ was considered statistically significant.

Prior to study initiation, the Michigan State

University Biomedical Institutional Review Board (MSU BIRB) and the University of Zambia, School of Medicine Biomedical Research Ethics Committee (UNZA BREC) provided ethical approval of the study. Written consent for participation was obtained from the participant or their proxy, if the participant was incapacitated.

\section{Results}

A total of 43 CHASE participants were imaged: 41 with CT and 2 using MRI. The mean age of participants was 37 years; 24 were male. Almost two-thirds had never received treatment with ARVs. Approximately $80 \%$ had advanced HIV infection (WHO Stage III or IV; mean CD4 count 186 cells $/ \mathrm{mm}^{3}$ ) and $44 \%$ had an OI identified. The neuropsychiatric symptom burden was high, with over half of participants endorsing symptoms of anxiety and depression severe enough to warrant further clinical evaluation based upon WHO recommendations using the SSQ.11 Over half also scored below normal values on the IHDS and on the less specific zMMSE suggesting a substantial burden of cognitive impairment in this cohort (Table 1).

Imaging abnormalities were identified in $70 \%$ of participants, with white matter abnor-

Table 1. Clinical characteristics and outcomes $(n=43)$.

\begin{tabular}{|c|c|}
\hline Characteristics & Values \\
\hline Age, mean years (SD) & $37.4(11.0)$ \\
\hline Gender, male n (\%) & $24(56)$ \\
\hline $\begin{array}{l}\text { CD4 count, mean (SD) } \\
\quad>200 \mathrm{n}(\%) \\
\quad<50 \mathrm{n}(\%) \\
50-200 \mathrm{n}(\%)\end{array}$ & $\begin{array}{l}186(215) \\
14(33) \\
12(28) \\
17(40)\end{array}$ \\
\hline WHO clinical stage, $n=40, n(\%)$ & $\begin{array}{l}\text { I: } 6(14) \\
\text { II: } 2(5) \\
\text { III: } 14(36) \\
\text { IV: } 18(43)\end{array}$ \\
\hline Antiretroviral use, n (\%) & $\begin{array}{l}\text { Never: } 24(56) \\
\text { Active: } 16(37) \\
\text { Defaulted: } 3(7)\end{array}$ \\
\hline $\begin{array}{l}\text { Psychiatric Symptom Burden n=59 } \\
\text { mean Shona Symptom Score (SD) }\end{array}$ & 4.6 (3.0): $56 \%$ requiring additional evaluation* \\
\hline $\begin{array}{l}\text { International HIV Dementia Scale, } \mathrm{n}=59 \\
\text { (mean, SD, \% impaired) }\end{array}$ & 8.7 (2.8): 54\% impaired \\
\hline $\begin{array}{l}\text { Zambian Mini-Mental State Exam n=59 } \\
\text { (mean, SD, \% impaired) }\end{array}$ & 20.7 (3.9): 51\% impaired \\
\hline Seizure severity at first seizure, $\mathrm{n}(\%)$ & $\begin{array}{l}\text { Single, brief seizure } 15 \text { ( } 35) \text {; Multiple seizures, } \\
\text { not status epilepticus } 20 \text { (47); } \\
\text { Status epilepticus } 8 \text { (19) }\end{array}$ \\
\hline CNS opportunistic infection $n=31, n(\%)$ & $14(44)$ \\
\hline Outcomes, n (\%) & $\begin{array}{l}\text { Recurrent seizures } 15 \text { (35) } \\
\text { Died } 9 \text { (21) }\end{array}$ \\
\hline
\end{tabular}

*Based upon score and recommendations during Zimbabwe-based validation studies. 
malities being the most common finding (56\%), primarily from vasogenic edema. Deep gray (19\%), posterior fossa (21\%), and cortical abnormalities (28\%) were also common. Contrast enhancement was seen in $29 \%$ of the 37 subjects who received contrast. Brain volumes were increased in $17 \%$ of subjects usually associated with CNS infections, while other participants showed evidence of generalized atrophy. Isolated enlarged ventricles, indicative of subcortical atrophy, were seen in $16 \%$ (Table 2). Given the prevalence of imaging abnormalities in this cohort, our analyses had $\sim 90 \%$ power to detect an effect size difference of $22 \%$ or more in the primary outcomes of interest (death and recurrent seizure). During follow-up, 15 (35\%) had recurrent seizures and 9 (21\%) died.

Patients with entirely normal brain images had a lower burden of depression and anxiety symptoms based upon the SSQ (3.0 vs. 5.3, $\mathrm{P}=0.04$ ) and patients with subcortical atrophy had a lower mean IHDS score (5.2 vs. 9.3, $\mathrm{P}=0.002)$. Although edema was associated with OIs (36 vs. 6\%, OR 8.79 (95\%CI: 1.03-236, $\mathrm{P}=0.04$ ), none of the imaging findings, including cortical lesions, were predictive of seizure recurrence or death (Table 3 ).

\section{Discussion and Conclusions}

In this cohort of HIV-positive patients with their first seizure, most had advanced HIV disease and status epilepticus was common.
Imaging abnormalities were evident in $70 \%$. The imaging findings were diverse and included evidence of acute OIs as well chronic atrophic changes. Recurrent seizures and death occurred in over half during follow-up. Structural brain lesions, evident primarily using CT, were not predictive of recurrent seizure or death in this cohort.

This patient population was evaluated at Zambia's only tertiary care referral center and as such they may not be representative of all adults with HIV in Zambia. Attempts to recruit from a community-based HIV clinic for more than 6 months failed to identify anyone with HIV presenting in the outpatient settings within 2 weeks of a new onset seizure. CT was the imaging modality used in 41/43 patients. MRI imaging may have identified lesions not evident on CT. However, CT is more readily available and less expensive in most African tertiary care centers and therefore this data may be particularly useful in such resource limited settings. Imaging was preferentially obtained on CHASE patients who had no evident etiology for their seizure after a thorough work up including extensive CSF studies. Recurrent seizures in participants who died may not have been adequately identified, but there was also no association between imaging abnormalities and death.

Participants with any abnormality on their brain image had a higher burden of psychiatric symptoms. This is particularly interesting since psychiatric morbidity, as measured by the SSQ, has previously been shown to predict early mortality in rural Zambians with HIV, even after controlling for HIV stage and socioeconomic status. 12 Subcortical atrophy was associated with clinical evidence of cognitive impairment based upon lower IHDS scores. Previous research in South Africa using MRI technology identified brain atrophy in several brain regions (white matter, thalamus, gray matter, subcortical regions) in HIV+ vs. HIVindividuals. ${ }^{8}$ Using CT technology, we found that subcortical atrophy was specifically associated with the evidence of lower mean scores on the IHDS but not zMMSE. Neuropsychiatric morbidity, including cognitive impairment, depression and anxiety, are likely underdiagnosed and undertreated in most resource limited settings. The association between psychiatric symptoms and structural brain lesions provides further support for the need to institute basic neuropsychiatric screening in HIV clinics. ${ }^{12,13}$ Epilepsy is a common non-communicable disease in SSA, where most people with HIV reside and the inevitable co-occurrence of two common conditions is further compounded by the high risk of provoked seizure in people with HIV due to OIs, metabolic derangements, ARVs which reduce the seizure threshold, and other challenges. Further studies are needed to determine neurologic vulnerabilities and outcomes in people with HIV, particularly in rural and pediatric populations. As HIV continues to evolve from a fatal to a chronic condition and neuroimaging becomes increasingly available, clinicians and researchers need additional epidemiologic and natural history data relevant to this population to direct care and set research priorities.

Table 2. Imaging abnormalities identified in HIV+ patients with new onset seizure $(n=43)$.

\begin{tabular}{|c|c|c|}
\hline Location of abnormality & Prevalence, n. (\%) & Description (n)* \\
\hline Entirely normal & $13(30)$ & - \\
\hline Cortical & $12(28)$ & $\begin{array}{l}\text { Decreased attenuation on CT/increased T2 on MRI (4); diffuse cortical thickening (1); focal } \\
\text { cortical thickening (2); focal cortical atrophy (1); multifocal enhancing lesions (1); } \\
\text { old CVA (1); small cortical defect (1); calcification (2) }\end{array}$ \\
\hline White matter & $24(56)$ & $\begin{array}{l}\text { Decreased attenuation on CT/Increased T2 on MRI: mild (3); markedly diffuse (5); } \\
\text { markedly multifocal (6). Gliosis (1); }{ }^{\circ} \text { focal vasogenic edema (8); focal deep infarct (old) (1) }\end{array}$ \\
\hline Deep gray matter & $8(19)$ & $\begin{array}{l}\text { Decreased attenuation on CT/Increased T2 on MRI with mass effect (3); } \\
\text { decreased attenuation on CT/Increased T2 on MRI without mass effect (2); } \\
\text { focal encephalomalacia (1); multifocal cystic (2) }\end{array}$ \\
\hline Posterior fossa & $9(21)$ & Decreased attenuation on CT/Increased T2 on MRI (6); edema (1); multifocal cystic (2) \\
\hline Contrast enhancement $(\mathrm{n}=34)$ & $10(29)$ & $\begin{array}{l}\text { Discrete cortical lesions (3); leptomeninges (4); vague peripheral (1); ring enhancing (1); } \\
\text { subcortical white matter and basil cisterns (1) }\end{array}$ \\
\hline Brain volume & $\begin{array}{l}\text { Atrophy } 7(16) ; \\
\text { normal } 29(67) ; \\
\text { mild swelling } 5(12) \\
\text { gross swelling } 2 \text { (5) }\end{array}$ & $\begin{array}{l}2=\text { atrophy; } 3=\text { normal; } 4=\text { mild swelling; } 5=\text { gross swelling. Decreased brain volume associated } \\
\text { with generalized atrophy }\end{array}$ \\
\hline Ventricular size & $\begin{array}{l}\text { Normal } 31(72) \\
\text { small } 5(12) \\
\text { large } 7(16)\end{array}$ & Increased ventricular size associated with subcortical atrophy \\
\hline
\end{tabular}




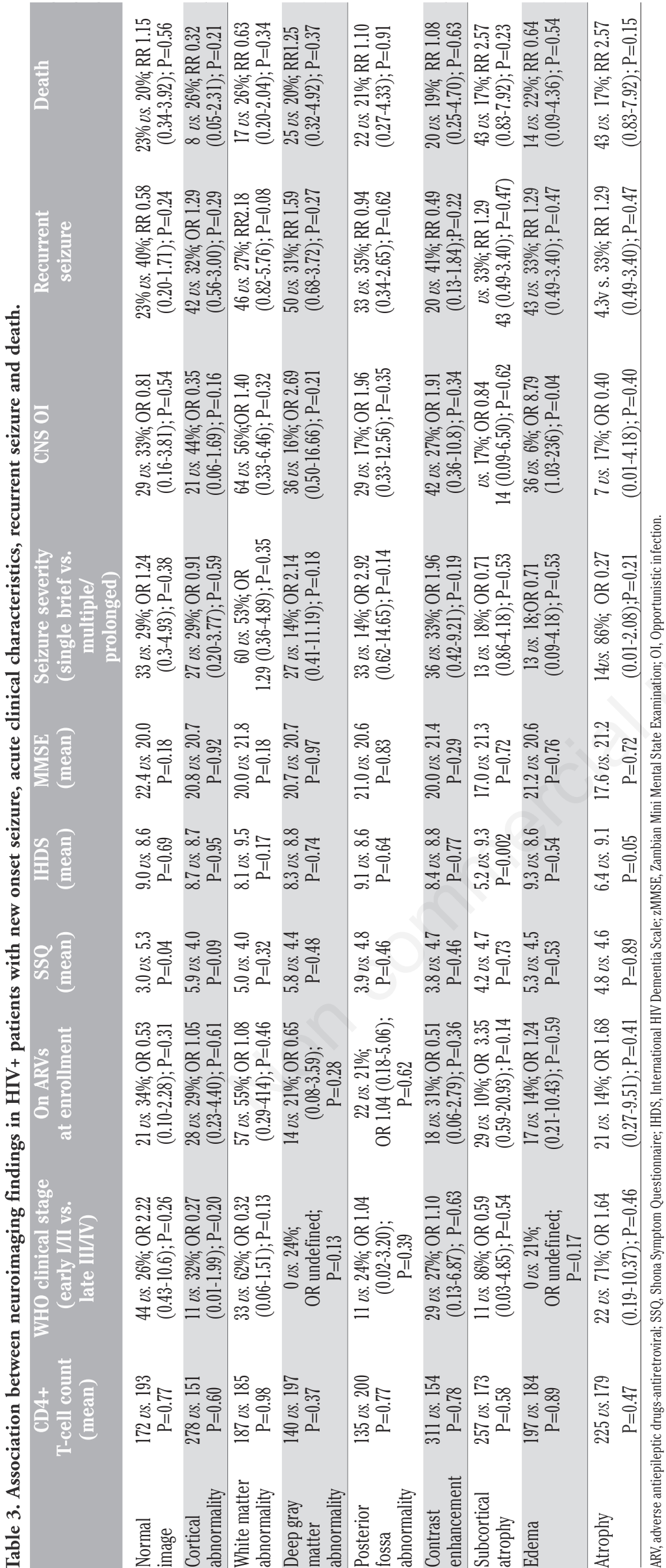

\section{References}

1. Krumholz A, Wiebe S, Gronseth G, et al. Practice parameter: evaluating an apparent unprovoked first seizure in adults (an evidence-based review): report of the Quality Standards Subcommittee of the American Academy of Neurology and the American Epilepsy Society. Neurology 2007;69:1996-2007.

2. Birbeck GL, French JA, Perucca E, et al. Evidence-based guideline: antiepileptic drug selection for people with HIV/AIDS: report of the Quality Standards Subcommittee of the American Academy of Neurology and the Ad Hoc Task Force of the Commission on Therapeutic Strategies of the International League Against Epilepsy. Neurology 2012;78:139-45.

3. Pesola GR, Westfal RE. New-onset generalized seizures in patients with AIDS presenting to an emergency department. Acad Emerg Med 1998;5:905-11.

4. Ortblad KF, Lozano R, Murray CJ. The burden of HIV: insights from the Global Burden of Disease Study 2010. AIDS 2013;27:2003-17.

5. WHO/WFN. Atlas: country resources for neurological disorders. Geneva: WHO; 2004. p 59.

6. Potchen MJ, Kampondeni S, Birbeck GL, et al. Magnetic resonance imaging in Malawi: contributions to clinical care, medical education and biomedical research. Malawi Med J 2011;23:60-4.

7. Wilmshurst JM, Burgess J, Hartley P, Eley B. Specific neurologic complications of human immunodeficiency virus type 1 (HIV-1) infection in children. J Child Neurol 2006;21:788-94.

8. Heaps JM, Joska J, Hoare J, et al. Neuroimaging markers of human immunodeficiency virus infection in South Africa. J Neurovirol 2012;18:1516.

9. Jelsma J, Mhundwa K, De Weerdt W, et al. The reliability of the Shona version of the EQ-5D. Central Afr J Med 2001;47:8-13.

10. Patel V, Simunyu E, Gwanzura F, et al. The Shona Symptom Questionnaire: the development of an indigenous measure of common mental disorders in Harare. Acta Psychiatr Scand 1997;95:469-75.

11. Patel V, Simunyu E, Gwanzura F, et al. The pathways to primary mental health care in high-density suburbs in Harare, Zimbabwe. Soc Psychiatry Psychiatr Epidemiol 1997;32:97-103.

12. Birbeck GL, Kvalsund MP, Byers PA, et al. Neuropsychiatric and socioeconomic status impact antiretroviral adherence 
and mortality in rural Zambia. Am J Trop Med Hyg 2011;85:782-9.

13. Kvalsund MP, Haworth A, Murman DL, et al. Closing gaps in antiretroviral therapy access: human immunodeficiency virusassociated dementia screening instru- ments for non-physician healthcare workers. Am J Trop Med Hyg 2009;80:1054-9.

14. Holguin A, Banda M, Willen EJ, et al. HIV-1 effects on neuropsychological performance in a resource-limited country,
Zambia. AIDS Behav 2011;15:1895-901.

15. Potchen MJ, Kampondeni SD, Ibrahim $\mathrm{K}$, et al. NeuroInterp: a method for facilitating neuroimaging research on cerebral malaria. Neurology 2013;81:585-8. 